\title{
Meta
}

Journal des traducteurs

Translators' Journal

\section{Préalables linguistiques pour une traduction assistée par ordinateur du système verbal arabe-français}

\section{Georges Dorlian}

Volume 42, numéro 2, juin 1997

Lexicologie et terminologie II (1) et Traduction et post-colonialisme en Inde

Translation and Postcolonialism: India (2)

URI : https://id.erudit.org/iderudit/002580ar

DOI : https://doi.org/10.7202/002580ar

Aller au sommaire du numéro

Éditeur(s)

Les Presses de l'Université de Montréal

ISSN

0026-0452 (imprimé)

1492-1421 (numérique)

Découvrir la revue

Citer cet article

Dorlian, G. (1997). Préalables linguistiques pour une traduction assistée par ordinateur du système verbal arabe-français. Meta, 42(2), 317-320.

https://doi.org/10.7202/002580ar
Résumé de l'article

Cet article vise à faire redécouvrir dans la structure verbale arabe, à un niveau profond, le système temporel propre à cette langue, système permettant l'élaboration d'une classification unifiée que les informaticiens pourront repenser et redéfinir d'une manière plus technique pour la mise en place d'un logiciel de traduction assistée par ordinateur. 


\title{
PRÉALABLES LINGUISTIQUES POUR UNE TRADUCTION ASSISTÉE PAR ORDINATEUR DU SYSTEME VERBAL ARABE-FRANÇAIS*
}

GEORGES DORLIAN

Université Libanaise, Tripoli, Liban

\begin{abstract}
Résumé
Cet article vise à faire redécouvrir dans la structure verbale arabe, à un niveau profond, le système temporel propre à cette langue, système permettant l'élaboration d' une classification unifiée que les informaticiens pourront repenser et redéfinir d'une manière plus technique pour la mise en place d' un logiciel de traduction assistée par ordinateur.
\end{abstract}

\begin{abstract}
This paper examines the tense system of the Arabic verb system. This will enable the development of a unified classification of Arabic verbs that computer specialists can use as a basis for defining, designing and implementing an Arabic-French computer-assisted translation software program.
\end{abstract}

Du fait même de leur simplicité et de leur réduction, les formes verbales arabes posent des problèmes quant à leur informatisation et — par la suite - quant à leur traduction en formes équivalentes dans d'autres langues. Déjà, la structure même du mot arabe, construit à base de racines consonantiques (trilitères ou quadrilitères) et infixes vocaliques (ces derniers ne sont pas reproduits au niveau graphique), posait et pose encore des difficultés quant à son éventuelle informatisation : faudra-t-il partir de la racine consonantique ou du mot voyellé pour former les classes de mots? Et puisque, à présent, ce qui nous intéresse c'est le système verbal et sa traduction en français dans l'optique de la mise en place d'un logiciel prévu à cet effet, nous concentrerons notre travail sur la correspondance des formes verbales de ces deux langues.

Il sera moins question dans le présent exposé du système verbal français - qui a fait et fait encore l'objet de travaux linguistiques avancés et orientés - que du système verbal arabe dont nous repérerons certains des aspects problématiques.

La linguistique descriptive et fonctionnelle avait depuis longtemps souligné la composante aspectuelle du système verbal arabe, lequel ne reconnaît que deux formes morphologiques dont les schèmes sont fa' al ('al-madi = l'accompli) et yaf' al ('al-mudari'

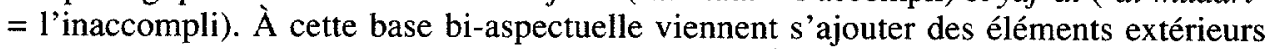
pour constituer l'éventail temporel et modal de l'arabe'.

L'arabe est resté à ce stade de la description de son système verbal, et aucune évolution n'a été signalée depuis le fameux Kitab de Sibawayhi (mort au VII' ${ }^{\mathrm{e}}$ siècle $=$ II $^{\mathrm{e}}$ siècle de l'hégire). Or, pour que s'opère un passage de l'arabe au français et inversement, certains obstacles sont à surmonter.

Le premier de ces obstacles émane du fait que, tandis qu'en français c'est le paradigme temporel (présent/passé) qui constitue le pivot stable, obligatoire et exclusif de la distribution verbale auquel viennent s'ajouter les morphèmes aspectuels (avoir / être + é $=$ l'accompli) facultatifs et discontinus, en arabe ce sont les aspects (accompli/inaccompli) 
qui constituent l'axe paradigmatique stable, et c'est l'expression du temps qui y est facultative. Ainsi apparaît une opposition incontournable quant à la position et à la distribution des éléments obligatoires et facultatifs dans chacune des deux langues en question.

Pour être plus clair, reprenons la règle de réécriture des formes verbales françaises telle que représentée par ce schéma qu'ont élaboré les générativistes français :

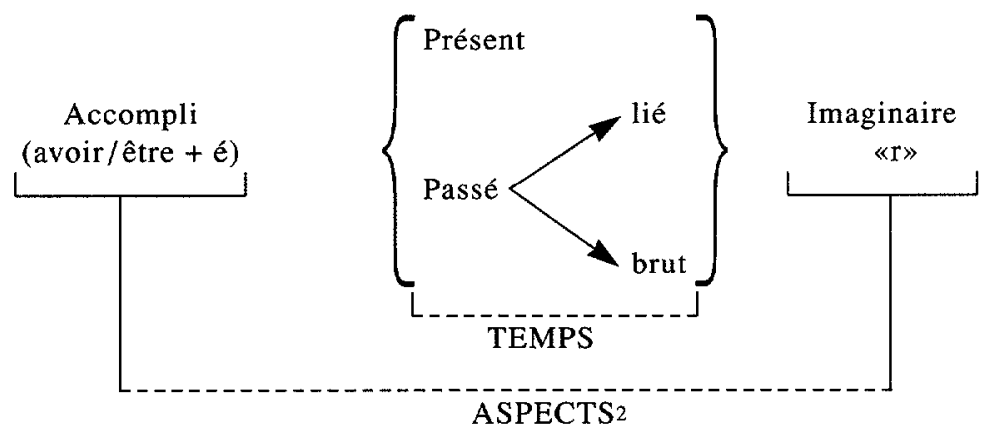

En français, c'est l'introduction du morphème de l'accompli par un segment discontinu «avoir/être + é» («avoir/être» se place en tête du SV et «é» à la fin du premier élément conjugable) dans le syntagme verbal qui crée les formes verbales à valeur accomplie. Nous avons ainsi un présent accompli (passé composé $=$ Prés. $+<$ avoir $/$ être $+e ́(V+V)$, un passé lié accompli (P.Q.P. $=$ «ais» $+<$ avoir / être + é $>+$ V), un passé brut accompli (Passé antérieur $=\langle a \mathrm{ai} »+\langle$ avoir $+\hat{e}\rangle+\mathrm{V}$ ), un futur accompli (Prés. $+\langle\mathrm{r} »+<$ avoir $/$ être $+\hat{e}\rangle+$ $V)$ et un conditionnel ou futur du passé accompli ( $\langle$ ais» $+\langle\mathrm{r} »+\langle$ avoir /être + é $\rangle+\mathrm{V})$.

En arabe, la distribution est toute différente. Le caractère accompli ou non accompli de l'expression verbale est déjà donné par la forme brute du verbe dans les schèmes $f a^{\prime} a l$ et yaf'al (et non par l'introduction d'un morphème équivalent au «avoir/être + é» du français), et c'est l'expression du temps qui se réalise par des éléments surajoutés, comme dans le schéma représentatif suivant:

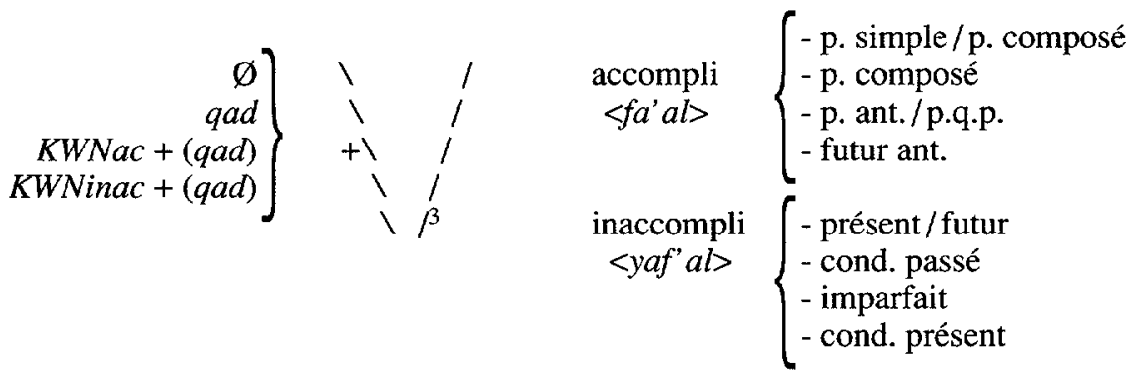

Un second obstacle se profile à travers la non-équivalence totale entre l'arabe et le français quant à l'expression du passé brut (passé simple) et du passé lié (imparfait) 
représentant respectivement l'énonciation historique et l'énonciation discursive. De désinences différentes en français (ai/ais), ces deux formes du passé font partie du paradigme stable des temps. En arabe, les choses sont différentes. Si le passé brut trouve son équivalent dans la forme fixe de l'aspect verbal accompli $\left(f a^{\prime} a l\right)$, le passé lié, quant à lui, révèle une construction composée : c'est l'accompli de l'auxiliaire $K W N$ (= kana) qui vient s'ajouter à la forme aspectuelle inaccomplie du verbe proprement dit (yaf'al). Il faudra mentionner à cet effet l'inexistence de la forme participiale en arabe : les deux constituants, auxiliaire et verbe, reçoivent une conjugaison qui, tout en étant différente dans certains cas (auxiliaire à l'accompli + verbe à l'inaccompli = imparfait), n'en est pas moins analogue.

Sur un autre plan, l'unicité de forme que requiert l'aspect accompli $\left(f a^{\prime} a l\right)$ rend impossible la distinction entre un passé brut historique et un accompli du présent lié au locuteur (respectivement le passé simple et le passé composé). Nous avons ici une situation inversée par rapport à la précédente. Tandis que l'imparfait arabe requiert un auxiliaire, le passé composé a la même forme brute que le passé simple. Aucune forme morphologique ne venant spécifier le passé composé, on s'en remet au sens de la phrase pour opérer la distinction ${ }^{4}$. Et souvent, l'interprétation de l'accompli fa' al est laissée au libre arbitre du traducteur, liberté entraînant parfois quelques anachronismes.

Enfin, la grammaire arabe ne reconnaît pas l'auxiliaire, ou tout au moins ne tranche pas sur la possibilité auxiliante ou copulative de certains verbes appelés incomplets ('af'al naqisa) ${ }^{5}$. «Incomplet» signifie que ces verbes ont besoin d'un complément pour parfaire le sens de la phrase. Nous avons là un cas analogue à celui du verbe copulatif français qui ne peut se suffire de son sujet et a besoin d'un autre syntagme (nominal, adjectival ou prépositionnel) pour constituer une phrase. Ainsi, un verbe incomplet comme $K W N$ (accompli = kana ; inaccompli $=y a k u n u$ ) est analysé comme élément verbal s'introduisant dans une phrase nominale et faisant du premier son sujet/agent ( $\left.{ }^{\circ} i s m u h u\right)$ et du second son prédicat (habaruhu). Cette solution, bien qu'elle soit arrangeante au niveau de surface, n'est pas satisfaisante pour peu qu'on veuille dépasser cette simple constatation superficielle et repenser la notion même de phrase nominale dans sa structure réduite à deux composantes nominales ( ${ }^{\circ} i s m=$ sujet $/$ agent, et habar $=$ prédicat) ou élargie par l'introduction d'un verbe dans l'une des deux composantes.

En effet, une phrase nominale simple comme ${ }^{\circ}$ al-waladu maridun (l'enfant <est> malade) est toujours interprétée - et partant traduite - comme un état se déroulant dans un présent continu. Avec l'introduction de kana (forme accomplie de la racine $K W N$ ), l'ensemble de l'état est renvoyé dans un passé continu et non-accompli et trouve sa traduction dans l'imparfait français. Cependant, on sait que l'équivalent de l'imparfait (ou de ce qu'on peut désigner par le passé continu et inaccompli) trouve sa réalisation en arabe dans la rencontre de deux verbes, l'un auxiliaire à l'accompli (kana) et l'autre à l'inaccompli (yaf'al). Cela nous permet de supposer la présence implicite et sous-entendue d'un verbe à l'inaccompli (yaf'al) dans la prétendue phrase nominale de surface. Cette supposition est d'autant plus vraie que dans une phrase nominale étoffée comme kana 'al-waladu yadhabu ${ }^{\circ}$ ila ${ }^{\circ}$ al-madrasati (l'enfant allait à l'école) - que l'analyse grammaticale arabe divise en trois parties : Kana $+{ }^{\circ} a l$-waladu (= sujet/agent de Kana) + yadhabu ${ }^{\circ}$ ila ${ }^{\circ}$ al-madrasati (proposition ayant la fonction de prédicat de kana) - , nous avons la présence explicite de deux verbes, Kana + yadhabu, où la rencontre de l'accompli de $K W N$ avec l'inaccompli du verbe $D H B$ donne le passé continu inaccompli, soit l'imparfait.

Cette reformulation de $K W N$ comme auxiliaire ou copule amène à une remise en question de deux notions : 1) la fonction de $K W N$ comme élément introducteur dans une phrase nominale se déplace vers le syntagme verbal (que ce dernier soit explicite ou implicite), il est donc un élément auxiliaire du verbe ; 2) la notion de phrase nominale est à repenser comme phénomène de surface, et de ce fait elle serait plutôt le résultat d'une 
transformation d'effacement du verbe, lequel est nécessairement présent dans la structure profonde.

Pour conclure, la démarche que nous venons d'exposer redécouvre dans la structure verbale arabe, à un niveau profond, le système temporel propre à cette langue, système permettant l'élaboration d'une classification unifiée que les informaticiens pourront repenser et redéfinir d'une manière plus technique pour la mise en place d'un logiciel de traduction assistée par ordinateur.

\section{Notes}

* Cet article est issu d'une communication présentée par l'auteur aux IVes Journées scientifiques du réseau «Lexicologie, terminologie, traduction» de l'AUPELF-UREF (Lyon, France, 28, 29, 30 septembre 1995).

1. Cohen, David (1989): L'aspect verbal.

2. Voir les travaux de J. Dubois (1966) : Syntaxe, Paris, Larousse.

3. Ce tableau est une reconstitution du système verbal arabe à partir du tableau de Cohen (1989): L'aspect verbal.

4. La particule qad ajoutée à la forme $f a^{\prime} a l$ est utilisée pour souligner et mettre en relief l'accomplissement, mais elle n'est pas une exigence impérative pour formuler l'accompli du présent. Voir à ce propos Cohen.

5. Voir l'article de Pierre Larcher sur l'auxiliaire arabe, CERLICO (1990), et l'article de Bohas et Ayoub sur la phrase nominale (1981). 